\title{
Dynamic Characteristics of Blade-Disk-Rotor System with Structural Mistuned Features
}

\author{
Chaofeng Li ${ }^{*}, 1$, Shihua Zhou ${ }^{1}$, Shuhua Yang ${ }^{2}$, Xiang Ren ${ }^{1}$, and Bangchun Wen ${ }^{1}$ \\ ${ }^{1}$ School of Mechanical Engineering \& Automation, Northeastern University, Shenyang 110819, P.R. China \\ ${ }^{2}$ The Research Institute, Shenyang Blower Works Group Co. Ltd, Shenyang 110819, P.R. China
}

\begin{abstract}
The finite element method is adopted to establish the dynamical models of blade, bladed disk and blades-diskshaft assemblies. Based on the analysis of mistuned structure and the dynamic characteristics of model in different levels, it gives the vibration mode distribution of different models. The research shows that the characteristics of the bladed disk and shaft have a huge difference, where some modes are caused by the strongly split and coupled vibration. The mistuned effects are likely to cause different coupled vibrations of blades between the blades-disk model and the blades-disk-shaft model. Meanwhile, it shows the frequency separation and concentration, and misses the system mode and the local vibration, which bring some difficulties for designing the blade-rotor system. In this paper, the results may provide a certain reference for blade-rotor system design and diagnosis.
\end{abstract}

Keywords: Blades-rotor, mistuned model, frequency separation, local vibration.

\section{INTRODUCTION}

The theoretical structure of axial-flow compressor blades-disk- shaft is a cyclic symmetric structure. Its dynamic behaviors have cyclic and symmetrical characteristics, and the eigenvalue of vibration equation also has some regularity. But in practical engineering, the bladesdisk-shaft system with uncoordinated phenomenon is due to the installation error, wear, requirement of dynamic balancing and installation technology, which is called mistuned structure. Generally, the mistuned structure easily causes the split of the local mode and the natural frequency. The dynamic analysis of the mistuned bladed disk is extensively studied by the international and domestic scholars in recent years, and the effects of the mistuned blades-disk could be better understood by those studies. In 1976, Ewins [1] studied the mistuned vibration mode of blades-disk structure firstly, and found the "complex modes" through the experiment. However, he did not realize that the phenomenon was the vibration modal localization. Vibration localization of bladed disk structure has an adverse effect on the high cycle fatigue, which causes the mistuning [2, 3]. There are three kinds of mechanical model of the mistuned bladed disk [4]: (1) the lumped parameter model, the model where every bladed disk segment is connected to the base of the single degree or multi-degree of the spring-mass oscillator, in order to simulate the coupling property between the blades, each oscillator is connected with the massless spring, and the effect of damping is considered in the coupling vibration of oscillator by some authors [5-7].

*Address correspondence to this author at the School of Mechanical Engineering \& Automation, Northeastern University, Shenyang 110819, P.R China; Tel: +86-024-83674491; Fax: +86-024-83674491;

E-mail: chf.li@mail.neu.edu.cn
(2) The continuous parameter model, to study the influence of the mistuned structure with different parameters, the blade is considered as a continuous parameter model $[8,9]$. (3) Through FE model, in 1982, Rzadkowski [10] analyzed the free vibration of mistuned bladed-shaft structure with the finite element model (FEM), which was relatively simple, but the simulation precision was low. The multi-level mistuned bladed disk mode was studied by Denis [11], which proved effective and considered multi-stage coupling mistuned characteristics of bladed discs. With the development of computer technology, the FEM method has made great progress. Using the FEM to study the vibration characteristics of mistuned system makes the results more accurate. These studies are mostly about model and reduction of the blades-disk circulation structure to study the coupling vibration characteristics of mistuned blades-disk model [12-17]. Rzadkowski and Kovalov [12] concluded that blade mistuning has stabilizing effects on bladed disks supported by a rigid shaft and flexible shaft. Yang [13] proposed a method to reduce freedom and calculated steadystate response of the mistuned bladed disk system, and the research showed that the method had a high fidelity, and greatly improved the computational efficiency. Ge [14] studied the method to assess the localization of mistuned blades-disk multistage dynamics. Castanier [15] studied random bladed disk response with contact interface characteristic. Based on model strain energy theory, the paper evaluates the localization factor mistuned multistage bladed disk vibration model characteristics. In turbine machinery, the study of the vibration characteristics of individual components such as blades, bladed disk and shaft has been established as an important part of the design. However, the vibration characteristic of an individual component can change considerably when these components 
are assembled together to form one system, due to the coupling effects among these constituent components. These variations destroy cycle symmetry.

This paper established the FEM of blades-disk-shaft system. Compared with the results of single blade model, mistuned bladed disk model, and mistuned blades-disk-shaft model, the relationships between the natural frequencies of three different systems are investigated based on critical speed, the frequency separation and coupling vibration mode caused by the mistuning effect and.

\section{THE MODEL OF BLADES-DISK-SHAFT}

\subsection{The Inherent Characteristics Comparison of Single Blade Model}

In order to comparatively analyze the dynamic characteristics of blade with different boundary structures, the single cantilevered blade needs to be analyzed first. The blade width is equal to $0.02 \mathrm{~m}$ and the thickness is $0.0025 \mathrm{~m}$, which has all constraints at the end. Blade model is divided into 160 units and 410 nodes, the natural frequencies and vibration modes of $0.250,0.225$ and 0.20 are calculated. In addition, the width of blade is $0.02 \mathrm{~m}$. The thickness is equal to $0.02 \mathrm{~m}$. The density is $8304 \mathrm{~kg} / \mathrm{m}^{3}$. The elastic modulus is equal to $2.069 \times 10^{11} \mathrm{~Pa}$. The Poisson's ration is 0.3 . The inherent characteristic is compared by using the analytic method (AM) and the FEM.

\subsubsection{Analytical Method (AM)}

It is assumed that the bladed disk is the rigid disk and neglects the strain energy of bladed discs. Based on the rotational speed of blade, according to the relevant knowledge of elastic mechanics and mechanics of materials, the kinetic energy and strain energy of blade can be described as:

$$
\begin{aligned}
& T_{b}=\frac{1}{2} \int_{r_{d}}^{r_{b}} \rho_{b} A_{b}\left\{\begin{array}{l}
\left(\frac{\partial v_{b}}{\partial t}\right)^{2}+\left[v_{b}^{2}+(x+R)^{2}\right] \Omega^{2}+ \\
2(x+R) \Omega \frac{\partial v_{b}}{\partial t}
\end{array}\right\} \mathrm{d} x+\frac{1}{2} J_{R} \omega \Omega^{2} \\
& U_{b}=\frac{1}{2} \int_{r_{d}}^{r_{b}} E_{b} I_{A}\left(\frac{\partial^{2} v_{b}}{\partial x^{2}}\right)^{2} \mathrm{~d} x \\
& +\frac{1}{4} \int_{r_{d}}^{r_{b}} \rho_{b} A_{b} \Omega^{2}\left[2 R\left(r_{b}-x\right)+r_{b}^{2}-x^{2}\right]\left(\frac{\partial v_{b}}{\partial x}\right)^{2} \mathrm{~d} x
\end{aligned}
$$

where, $v_{\mathrm{b}}$ is the speed in arbitrary position of blade in $y$ direction, $I_{\mathrm{A}}$ represents the section inertia of blade and $J_{\mathrm{A}}$ is the inertia of bladed disk.

Using the equation discretization of Leeds Galerkin method, and the Leeds Galerkin expansion can be expressed as:

$$
u(x, t)=\sum_{i=1}^{n} \phi_{i}(x) q_{i}(t)=\mathbf{U}^{\mathbf{T}} \mathbf{q}(t)
$$

where, $\phi_{i}(x)$ is the shape function of the $i^{\text {th }}$ order, $q_{i}(x)$ represents generalized coordinate.

$\mathbf{U}=\left[\phi_{1}, \phi_{2}, \phi_{3}, \cdots, \phi_{n}\right]^{\mathrm{T}}$

$\mathbf{q}(t)=\left[q_{1}(t), q_{2}(t), q_{3}(t), \cdots, q_{n}(t)\right]^{\mathrm{T}}$

Substituting the values of Eq. (4) into the equations (1) and (2) yields are:

$$
\begin{aligned}
T_{b} & =\frac{1}{2} \dot{\mathbf{q}}^{T} \mathbf{M} \dot{\mathbf{q}}+\frac{1}{2} \Omega^{2} \mathbf{q}^{T} \mathbf{M q}+\frac{1}{2} \rho_{b} A_{b} \int_{r_{d}}^{r_{b}} x^{2} \Omega^{2} \mathrm{~d} x \\
& +\rho_{b} A_{b} \int_{r_{d}}^{r_{b}} x \Omega \mathbf{U}^{T} \mathrm{~d} x \dot{\mathbf{q}} \\
U_{b} & =\frac{1}{2} \mathbf{q}^{T} \mathbf{K} \mathbf{q}+\frac{1}{2} \Omega^{2} \mathbf{q}^{T}\left(\mathbf{K}_{\boldsymbol{\Omega} 1}-\mathbf{K}_{\boldsymbol{\Omega} 2}\right) \mathbf{q}
\end{aligned}
$$

According to the Lagrange equation, the energy and virtual work of rotating blade is:

$$
\mathbf{M} \ddot{\mathbf{q}}+\mathbf{C} \dot{\mathbf{q}}+\mathbf{K q}=\mathbf{F}
$$

where, $\mathrm{F}$ is the generalized rubbing force, $\mathrm{Fc}$ is the generalized damping force.

$$
\begin{aligned}
& \mathbf{M}=\rho_{b} A_{b} \int_{0}^{r_{b}} \mathbf{U} \mathbf{U}^{\mathbf{T}} \mathrm{d} x, \mathbf{K}=\mathbf{K}_{e}+\Omega^{2}\left(\mathbf{K}_{\mathbf{\Omega}}-\mathbf{K}_{\mathbf{\Omega} 2}-\mathbf{M}\right) \\
& \mathbf{K}_{e}=E_{b} I_{A} \int_{0}^{r_{b}} \mathbf{U}^{\prime \prime} \mathbf{U}^{\prime \prime \mathbf{T}} \mathrm{d} x \\
& \mathbf{K}_{\mathbf{\Omega} \mathbf{1}}=\frac{1}{2} \rho_{b} A_{b} r_{b}^{2} \int_{0}^{r_{b}} \mathbf{U}^{\prime} \mathbf{U}^{\prime \mathbf{T}} \mathrm{d} x \\
& \mathbf{K}_{\mathbf{\Omega} 2}=\frac{1}{2} \rho_{b} A_{b} \int_{0}^{r_{b}}\left[2 R\left(r_{b}-x\right)-x^{2}\right] \mathbf{U}^{\prime} \mathbf{U}^{\prime \mathbf{T}} \mathrm{d} x
\end{aligned}
$$

Natural frequencies of different length blades can be calculated by matrix and stiffness matrix of Eq. (7), which provides the basis for comparison.

\subsubsection{The Finite Element Model and Comparison}

It applies the finite element software ANSYS to analyze the inherent characteristics of single blade model and uses the solid 45. The single blade is divided into 160 elements with 410 nodes as shown in Fig. (2a).

By applying the AM and the FEM, the natural frequencies and vibration modes of blade are shown in Table 1 (In this table, $\mathrm{CB}$ represents the mode of circumferential bending, AB represents the mode of Axial bending, $\mathrm{T}$ represents the mode of torsion). Due to the limitations of the analytical model, $1^{\text {st }} \mathrm{AB} 1^{\text {st }} \mathrm{T}$ cannot be calculated. It can be seen from Table 1 that the three orders model results show a good agreement. The maximum error is $2.81 \%$. Therefore, theoretic analysis and simulation results show that the FEM has a high accuracy, which shows the first 5 vibration modes of three kinds of blades (the first circumferentially bent, second circumferential bent, first order buckling, third circumferential bent, first torsional bent) and the corresponding eigenvalue. The first 5 vibration modes are shown in Fig. (1). Compared to the first 5 vibration modes of 
Table 1. The first 5 natural frequencies of single blade.

\begin{tabular}{|c|c|c|c|c|c|}
\hline \multirow{2}{*}{$\begin{array}{c}\text { Blade } \\
\text { Length }(\mathbf{m})\end{array}$} & The First & The Second & The Third & The Fourth & The Fifth \\
\cline { 2 - 6 } & AM/FEM & AM/FEM & AM/FEM & AM/FEM & AM/FEM \\
\hline \hline 0.250 & $33.35 / 32.44$ & $209.05 / 203.48$ & $-/ 257.78$ & $585.35 / 571.33$ & $-/ 771.33$ \\
\hline 0.225 & $40.04 / 40.07$ & $250.9 / 251.29$ & $-/ 317.90$ & $702.6 / 705.54$ & $-/ 858.80$ \\
\hline 0.200 & $50.68 / 50.74$ & $317.59 / 318.17$ & $-/ 401.74$ & $889.3 / 893.22$ & $-/ 968.67$ \\
\hline Modes & $1^{\text {st }} \mathrm{CB}$ & $2^{\text {nd }} \mathrm{CB}$ & $1^{\text {st }} \mathrm{AB}$ & $3^{\text {rd }} \mathrm{CB}$ & $1 \mathrm{st} \mathrm{T}$ \\
\hline
\end{tabular}

three types of blades, it can be found that the natural frequencies increase with shorter blade lengths, and with the increase in order, the amount of natural frequency increases. In order to obtain the corresponding effects more easily, a slightly larger mistuned value is adopted in this paper. (a)

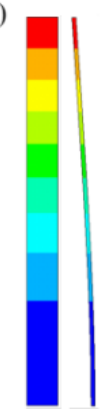

(b)

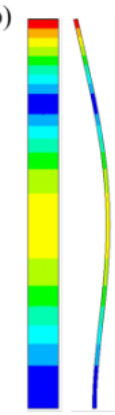

(c)

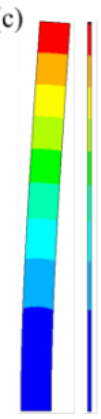

(d)

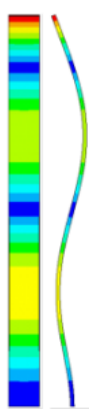

(e)

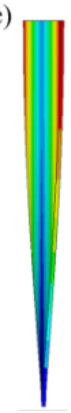

Fig. (1). The first 5 modal shapes of single blade.

\subsection{The FE Model of the Blades-Disk-Shaft Assembly}

As shown in Fig. (2), the mistuned model consists of single blade, blades-disk and blades-disk-shaft assembly. For the mistuned blade, the mistuned length of blade is the focus of attention, and the blades are divided into three levels (I, II, III). The other main parameters are as follows: the lengths of the shaft is equal to $2.5 \mathrm{~m}$, and the diameter is $0.1 \mathrm{~m}$. the inner diameter, outer diameter and the thickness are equal to $0.1 \mathrm{~m}$, $0.7 \mathrm{~m}$ and $0.021 \mathrm{~m}$ respectively.

\section{THE NATURAL CHARACTERISTIC OF THE MISTUNED MODEL}

The distributions of the mistuned vibration model are shown in Figs. $(3,4)$, which consist of natural frequencies, vibration modes and nodal diameter information. In addition, it also shows the coupling characteristics of vibration mode, the number of frequency and the vibration characteristic, such as bending vibration mode and the nodal diameter number.

The nomenclatures of Figs. $(\mathbf{3}, \mathbf{4})$ are shown in Table 2. In addition, the symbols in front of the vibration mode represent the mode characteristics, such as the $\mathrm{n}$ nodal diameter or $\mathrm{n}$ order bending, the number in the bracket represents the occurrence number. Several symbols of vibration mode appeared simultaneously, which represent the coupling vibration model; the first is the main vibration model, and others are the weaker ones. Each horizontal axis indicates a model, and the arrow is the direction of frequency increase. The circle on each shaft represents the first order natural frequency, the dotted box with inclined section lines represents the transformation of three-level model frequency, and the dotted box with cross section lines represents the transformation of two-level model frequency (the bladesdisk mode and blades-disk-shaft mode). The frequency with deficiency rectangle frame represents the rotational frequency, and the frequency with deficiency ellipse represents the new frequency.

In Figs. $(3,4)$, all modes of three kinds of blades are shown on single blade shaft (Table 1). For the single blade of the first order circumferential bending, the frequency may decrease slightly because of the flexible disk effect, and the frequency split takes place, but it is not obvious. The blade frequencies are divided into 9,8 and 7 (corresponding to the number of all kinds of blades respectively). The first and second orders circumferential bending modes also decrease slightly due to the effect of flexible disk, and the frequency split is not obvious. Meanwhile, for the first, the second and

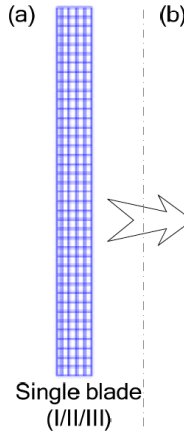

(b)

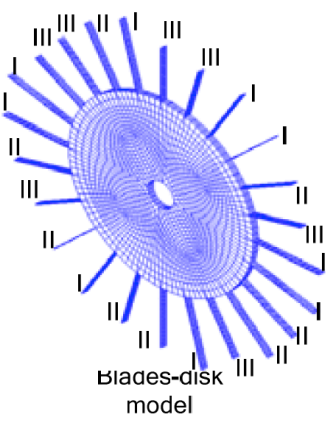

(c)

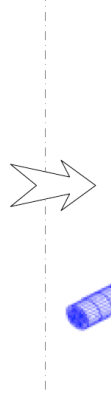

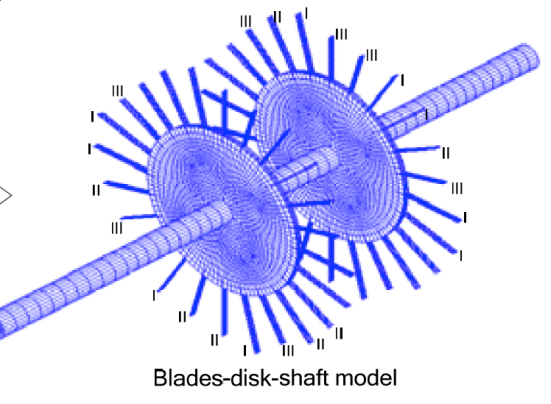

(d)

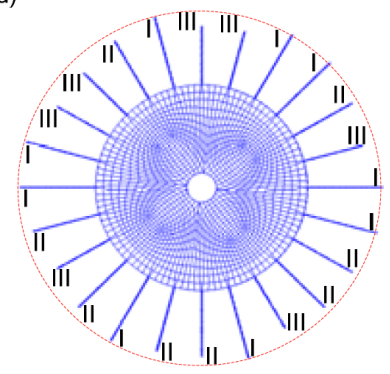

Blades-disk model the left view

Fig. (2). The FE model of the mistuned blades-disk-rotor system. 
Table 2. Vibration mode symbol.

\begin{tabular}{|c|c|c|c|c|c|c|}
\hline Sign & (a) & $\Lambda$ & $\theta$ & 6 & 㽞 & (8) \\
\hline Implication & pitch circle of disk & bending of shaft & nodal diameter of disk & circumferential bending & axial bending & torsion \\
\hline
\end{tabular}

the third circumferential bending modes, the frequencies split are obvious. For the first order axial bending model of blade, the frequency split of third kinds of blades become obvious, but the frequency split number is not equal to the blade number with the exception of the axial bending modal of the third blade. However, the coupled axial bending vibration modes appear between type II and type III. The frequency of torsional vibration is same as the previous first order circumferential bending vibration, which has no obvious split and lower frequency.

As it is shown in Fig. (3), the first order circumferential bending of the blade decreased compared to the blades-disk one due to the effect of shaft flexibility, but it has no obvious change. For the second order and third order circumferential bending modes of the blade, the obvious phenomenon of frequency increment and frequency split is affected by the shaft flexibility. For the first order axial bending of the blade, the characteristics of frequency split are kept invariant. Besides the frequency increment of blade III (382.80 400.046Hz), the first order circumferential bending and bladed disk mode remain consistent with the other two blades, the second order axial bending of blade III goes with the first order axial bending of blade II. For the torsional vibration model of the blade, it can be found that the frequency split becomes obvious because of the shaft flexibility.

The coupling vibration of the bladed disk and blade is affected by the shaft flexibility, such as the coupled vibration

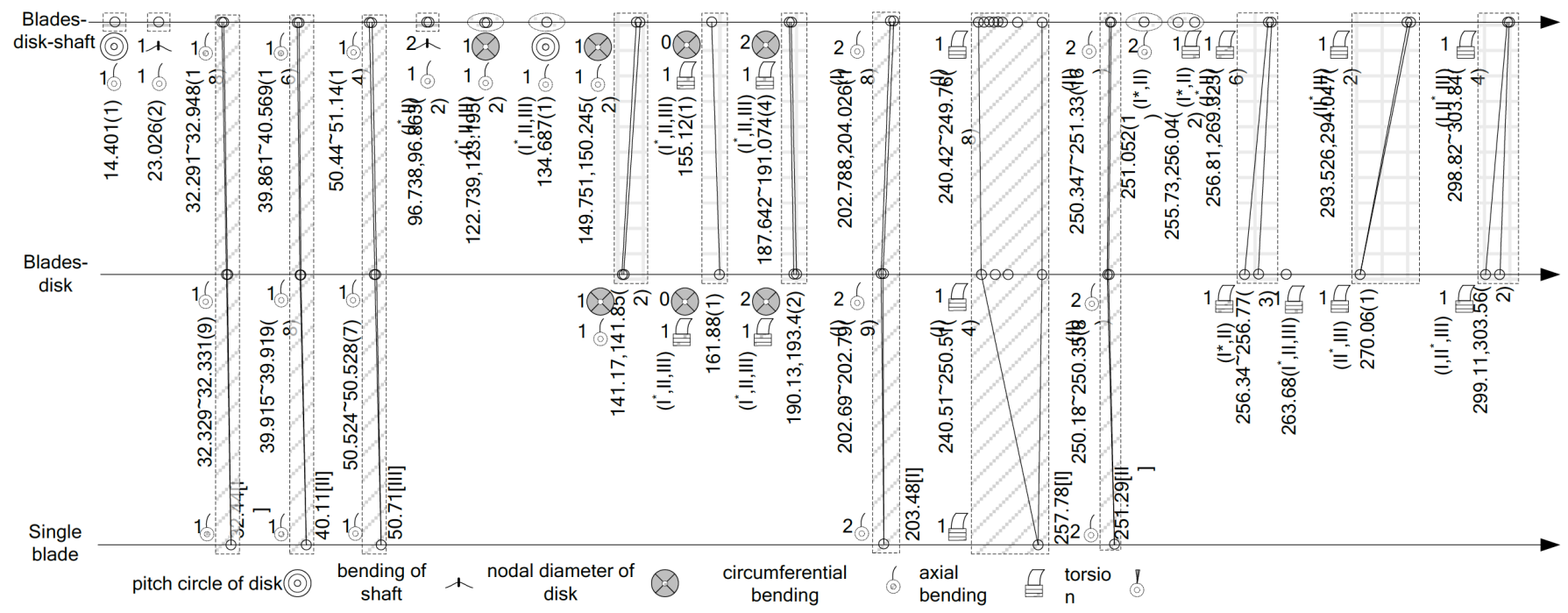

Fig. (3). The distribution of mistuned mode (I).

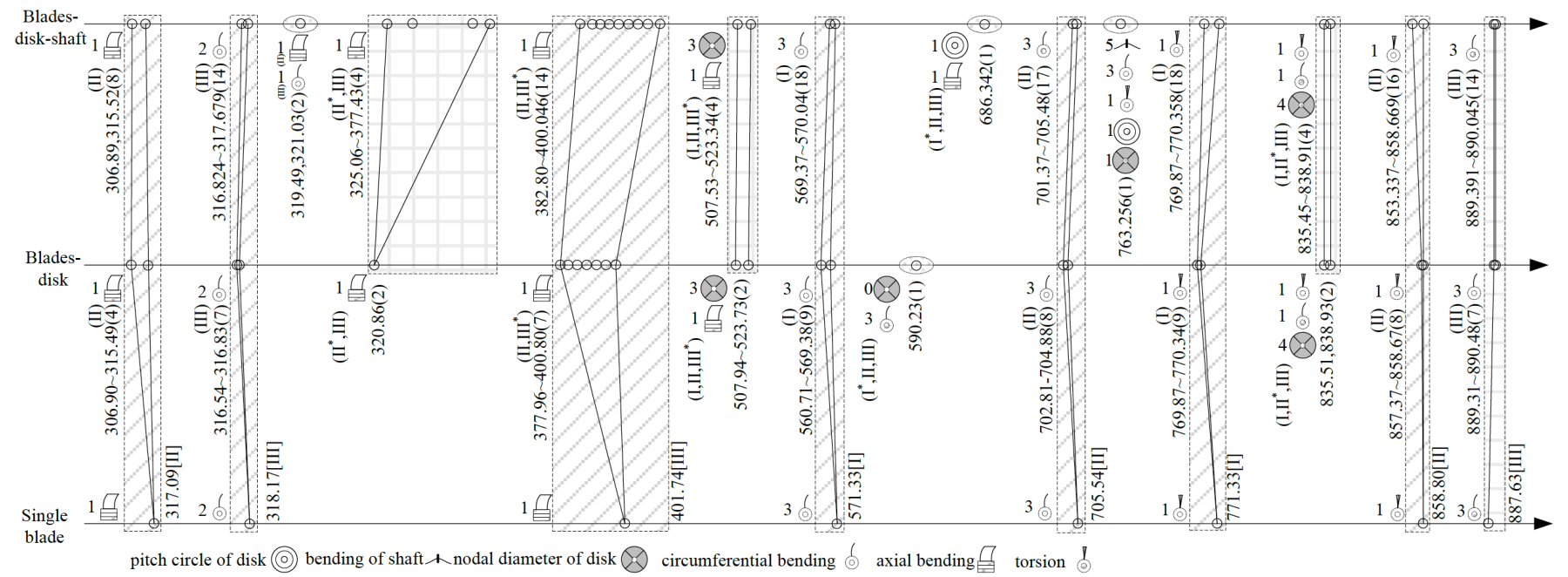

Fig. (4). The distribution of mistuned mode (II). 
of the one nodal diameter of bladed disk and the first order circumferential bending $(141.17 \mathrm{~Hz})$ of blade I, the coupling vibration of the zero nodal diameter of bladed disk and the first order axial bending of blade I (blade I is the main mode, $161.88 \mathrm{~Hz}$ ), and the coupling vibration of the first order circumferential bending, the first torsion and the zero nodal diameters of blade III $(835.51 \mathrm{~Hz})$ etc. In addition to the coupling vibration between disk and blade, the axial bending vibration of the different type of blades appear, such as coupling vibration of main axial vibration of blade I with auxiliary axial vibration of blade II, and the coupling vibration of main axial vibration of blade II with auxiliary axial vibration of blade III. For $(270.06 \mathrm{~Hz}, 320.86 \mathrm{~Hz})$ and $(299.11 \mathrm{~Hz}, 303.56 \mathrm{~Hz})$, the coupling features are the same, but with larger differences in frequency.

The coupling vibration of disks and blades in the bladedisk-shaft model are all reflected with the effects of shaft, and there is no obvious regularity in the frequency. The coupling vibration of the blade in bladed-disk model shows increase in modal characteristic values, and the separation phenomenon of the other blade coupling axial vibration frequency values slightly decrease and appear as a centralized tendency except for the $320.86 \mathrm{~Hz}$ of blade coupling vibration. It should be pointed out that not all frequency values in the blades-disk-shaft model can be found corresponding to the blade-disk coupling vibration due to the effects of the shaft, which is called "losing frequency" of the phenomenon. As priority is given to the dial 0 nodal diameter and blade III of $3^{\text {rd }}$ order circumferential bending (type I mainly, $268.68 \mathrm{~Hz}$ ) coupled vibration, the bladesdisk-shaft model does not exist in the corresponding frequency values. It can also be found that the vibration characteristics of the blade III $(377.96 \sim 400.80 \mathrm{~Hz})$ axial value increase significantly.

Besides the vibration mode reflecting the vibration of blade and disk, it appears that the model characteristics contain the axial vibration on the blade-disk-shaft due to the effects of the shaft. All the forms of coupling vibration show that due to the torsional rigidity of shaft, the vibration of rotary pitch circle and first order bending coupling vibration of the blade appear at a low frequency $(14.401 \mathrm{~Hz})$, and the first order bending vibration of the blade and the vibration of the blade and the shaft vibration coupling modes appear at $23.026 \mathrm{~Hz}$. The first order bending vibration of shaft and first order bending coupling vibration of the blade I and the blade II nearly appear at $96.738 \mathrm{~Hz}$, and 5 order bending of shaft, third order circumferential bending of blade, first order reverse and the coupling vibration of rotary pitch diameter and the pitch circle appear at $763.256 \mathrm{~Hz}$. For the $3 \sim 4$ orders bending vibration of shaft, the corresponding frequencies are not found in the mistuned model, which belongs to the "throw frequency". In addition, due to the coupling of mistuned blades-disk-rotor system, it also appears in a number of frequencies such as $(122.739 \mathrm{~Hz}, 134.687 \mathrm{~Hz}$, $251.025 \mathrm{~Hz}, 255.73 \mathrm{~Hz}, 319.49 \mathrm{~Hz}$ and $686.342 \mathrm{~Hz}$ ) which are shown on the axes of blades-disk-rotor system in Figs. $(\mathbf{3}, \mathbf{4})$. The new frequencies behave as the coupling vibration between disk and three types of blades, and between the three types of blades. Besides the coupling vibration of blades and disk, the vibration model of single blade also appear.

\section{THE RESULT ANALYSIS AND SUPPLEMENT}

According to the contrast calculation of mistuned model, because of the blade mistuned characteristics, the different types of blades appear in different vibration modes in the mistuned model. For the blade circumferential bending and the torsional vibration, the law of modes are the same as mistuned models due to the effects of disk and blade. The modes order and the corresponding blades number are equal due to the effects of the disk. The shaft causes double blade number order mode. However, there is no obvious relationship among the corresponding blade numbers. The blades are divided into 4 order modes at $257.78 \mathrm{~Hz}$ of blade I and at $317.09 \mathrm{~Hz}$ of blade II; but due to the effects of the disk, the shaft only causes 2 order modes. For the first order axial bending of blades III, it can be found from Fig. (4) that the number of frequency separation is equal to the number of blades. For the mistuned model, the disk and shaft have a relatively larger effect on the blade axial bending vibration, which is found in the modal concentration. In addition, the frequency values appear in the vibration model that the blade axial bending is the main model, and the disk 2 pitch diameter is supplementary, which is shown in Fig. (5). In fact, it causes the model localization. The first order mode of blades I at $240.51 \mathrm{~Hz}$ is shown in Fig. (6), it can be seen that it appears at the locational vibration obviously. In addition, it can be seen from Figs. $(3,4)$ that the mistuned characteristics make the new frequencies of blades-disk-shaft increased, and appear mostly in high frequency position. Meanwhile, it makes the coupling modes increased between blades-disk and blades, but the coupling modes of the blades-disk-shaft model increased. (a) Isometric view

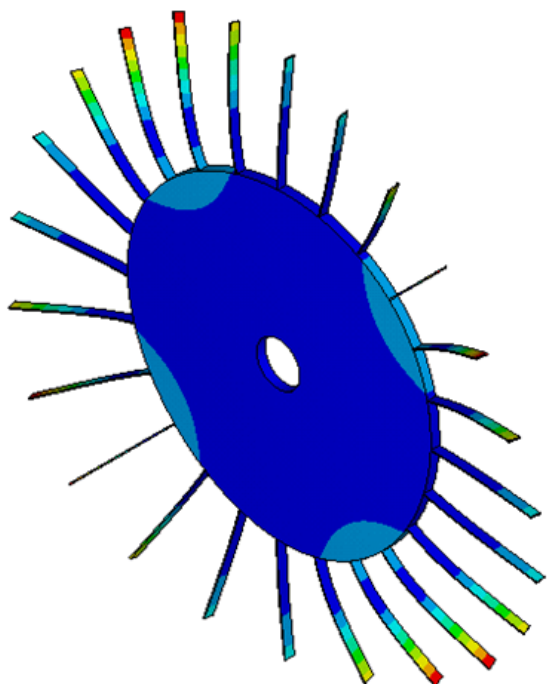

(b) Side views

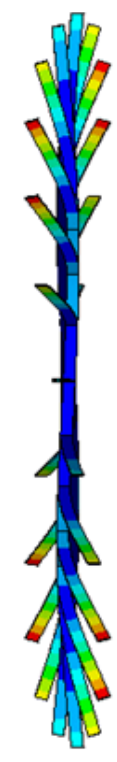

Fig. (5). A coupled mode of blade axial bending vibration. 
(a) Isometric view

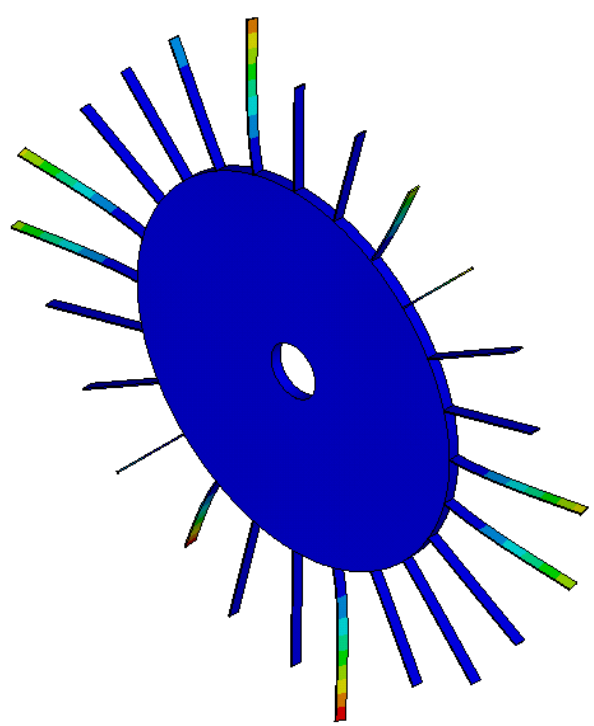

(b) Side views

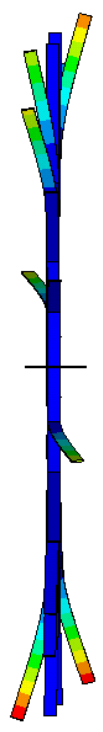

\section{ACKNOWLEDGEMENTS}

The work was financially supported by China Natural Science Funds (No. 51105063) and the Fundamental Research Funds for the Central Universities (No. N120403004).

\section{REFERENCES}

[1] D. J. Ewins, "Vibration modes of mistuned bladed disk", $A S M E J$ Eng. Power, vol. 98, no.7, pp. 349-355, 1976.

[2] J. J. Wang, Q. H. Li, and Z. G. Zhu, "Vibratory localization of mistuned bladed disk assemblies", Adv. Mechan., vol. 30, no. 4, pp. 517-528, 2000

[3] M. P. Castanier, and C. Pierre, "Modeling and analysis of mistuned blade disk vibration: current status and emerging directions", $J$. Propul. Power, vol. 22, no. 2, pp. 384-396, 2006.

[4] J. J. Wang, J. D. Xu, and Q. H. Li, "Analytical models of mistuned bladed disk assembles-a review", Turb. Technol., vol. 5, no. 4, pp 256-259, 2004

[5] C. C. Lin, and M. P. Manolete, "Effects of damping and damping scheme for the analysis of mistuning on the forced vibration response of bladed disks", J. Sound Vib., vol. 13, no. 2, pp. 153$160,1996$.

[6] C. C. Lin, and M. P. Manolete, "An adaptive perturbation scheme for the analysis of mistuning on the forced vibration response of bladed disks", ASME J. Eng. Power, vol. 11, no. 9, pp. 153-160, 1997.

Fig. (6). The vibration mode localization of type I blade.

\section{CONCLUSION}

[7] H. Y. Hong, Y. K. Jae, and J. I. Daniel, "Vibration localization of simplified mistuned cyclic structures undertaking external harmonic force", J. Sound Vib., vol. 261, no. 5, pp. 859-870, 2003.

The mistuned structure analysis discusses the three models of blade, blades-disk model and blades-disk-shaft model in the paper, which gives the corresponding modes distribution diagram. The following conclusions are drawn:

Because of the effects of the disk flexibility, the model of blades-disk appears as coupling vibration, which is caused by different types of blades. With the effect of disk, the vibration frequency value of the coupling axial vibration of blade appears separated and concentrated, and the eigenvalues have greatly improved. Due to the mistuned blade structure, the 3 and 4 order bending vibration model of shaft disappear, and lost frequency phenomenon and local model concentration appear in the blades-disk model and blades-disk-shaft model.

The mistuned structure makes the frequency distribution become more continuous. It is difficult to design the rotating blade rotor system. So the effect of blade mistuned quantity on the system should be further studied.

\section{CONFLICT OF INTEREST}

The authors confirm that this article content has no conflict of interest.
[8] S. T. Jeffrey, J. H. Joseph, and W. G. Robert, "Vibration of mistuned bladed-disk assembly using structurally damped beams", AIAA J., vol. 36, no. 12, pp. 2225-2228, 1998.

[9] B. W. Huang, and J. H. Kuang, "Mode localization in a rotating mistuned turbo disk with Coriolis effect", Inter. J. Mechan. Sci. vol. 43, no. 7, pp. 1643-1660, 2001.

[10] P. Rzadkowski, "Transient nozzle excitation of mistuned blades discs", J. Sound Vib., vol. 190, no. 4, pp. 629-643, 1996.

[11] L. Denis, and C. Pierre, "Modelling and analysis of multi-stage systems of mistuned bladed disks", Comput. Struct., vol. 89, no. 4 , pp. 316-324, 2011.

[12] R. Rzadkowski, and A. Kovalov, "2D in viscid flutter of the mistuned fourth and first stand configuration", Trans. Inst. Mach, vol. 112, no. 3, pp. 139-154, 2003.

[13] M. T. Yang, and J. H. Griffen, "A reduced order approach for the vibration of mistuned bladed disk assemblies", J. Eng. Gas Turbines Power, vol. 119, no. 1, pp. 161-167, 1997.

[14] C. C. Ge, J. J. Wang, and Y. Q. Liu, "Quantitative assessment method of vibration mode characteristics for mistuned multistage blisk", Aero, vol. 38, no. 1, pp. 25-29, 2012. modeling technique for mistuned bladed disks", J. Vib. Acoust., vol. 119 , no. 5, pp. 439-447, 1997.

[16] Z. J. He, B. I. Epureanu, and C. Pierre, "Fluid-structural coupling effects on the dynamics of mistuned bladed disks", AIAA J, vol. 45, no. 3, pp. 552-561, 2007.

[17] R. Bladh, M. P Castanier, and C. Peirre, "Reduced order modeling and vibration analysis of mistuned bladed disk assemblies with shrouds", J. Eng. Gas Turbines Power, vol. 121, no. 9, pp. 515522,1999 .
[15] M. P. Castanier, G. Ottarsson, and C. Pierre, "A reduced order

\section{(C) Li et al.; Licensee Bentham Open}

This is an open access article licensed under the terms of the Creative Commons Attribution Non-Commercial License (http://creativecommons.org/licenses/ by-nc/4.0/) which permits unrestricted, non-commercial use, distribution and reproduction in any medium, provided the work is properly cited. 